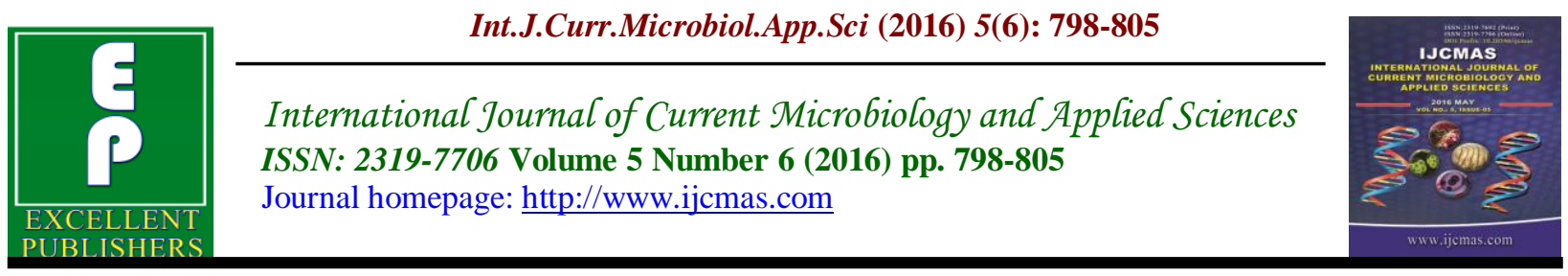

Original Research Article

http://dx.doi.org/10.20546/ijcmas.2016.506.089

\title{
Secular Trend of Antibiotic Resistance in Blood Stream Infections-A retrospective Analysis
}

\author{
Shweta Bohra*, Anamika Vyas, Mrityunjay Kumar and A. S. Dalal \\ Department of Microbiology, Geetanjali Medical College and Hospital, Udaipur, \\ Rajasthan, India \\ *Corresponding author
}

Keywords

Blood Stream Infections (BSIs), Antibiotic Resistance, CoNS.

\begin{tabular}{l}
\hline Article Info \\
\hline Accepted: \\
25 May 2016 \\
Available Online: \\
10 June 2016
\end{tabular}

\section{A B S T R A C T}

Bloodstream infections (BSIs) are among the most serious infections acquired by hospitalized patients requiring intensive care. The existence of a pathogen population with an ever-increasing resistance to antibiotics has complicated the clinical problems. This retrospective study was undertaken with a view to compile and analyse the common isolates of BSIs along with resistance pattern in a tertiary care teaching hospital. Retrospective study was conducted to identify the microbial profile in the blood culture isolates and their antibiotic susceptibility patterns in a tertiary care teaching hospital. The reports of specimens submitted for blood culture during the period of 2012-2015 to the microbiology laboratory were obtained, the positive cultures were identified, and data on the microbial species and their antibiotic patterns were collected and statically analysed. There were 4964 blood culture samples, of which 543 were identified to be culture positive. Of the total culture positives 177 (32.59\%) were Gram positive bacteria; 309 (56.91\%) were gram negative bacteria and $57(10.50 \%)$ were Candida species. Among the gram positive bacteria150 (27.62\%) were Coagulase negative Staphylococci (CoNS); 16 (2.94\%) were Staphylocococcus aureus and 11 (2.02\%) were Enterococcus species. In the Gram negative bacteria E.coli was 140 (25.78\%); Klebsiella pneumonia was 92 (16.94\%); Acinetobacter species was 25 (4.60\%); Pseudomonas specieswas32 (5.89\%). Statically significant resistance was observed in CoNS for Oxacillin; in E.coli for Ciproflaxacin, Amoxicillin-clavulanic acid, Piperacillin + tazobactam, Cefuroxime; and in Klebsiella pneumoniae for Amoxicillin-clavulanic acid, Piperacillin+tazobactam, Imipenem. CoNS is the common gram positive isolates followed by E.coli and Klebsiella pneumoniae in gram negative organisms of BSI in our setup. Significant resistance was observed for third generation cephalosporins, fluoroquinoles and piperacillin +tazobactam combination. Ongoing surveillance for antimicrobial susceptibility remains essential in case of BSI and will enhance efforts to identify resistance and attempt to prevent its spread.

\section{Introduction}

One of the major global problems is the rising trend in antibiotic-resistance mainly in hospitals, and also in the community which is a difficult condition to control without considerable measures and resources. The consequences of increased drug resistance are by far-reaching beyond any doubt when 
it is concerned with Blood stream Infection (BSI) making antibiotic resistance an important health issue. In many countries antimicrobial resistance among bloodstream pathogens have severe consequences including increased health care cost, morbidity and mortality. This is especially true in countries like India, where, antibiotics are used extensively and considerable resistance is reported from all over the country.

BSI is potentially life-threatening condition and requires rapid identification with the antibiotic susceptibility pattern of the causative agent in order to facilitate specific antimicrobial therapy. Despite the availability of newer antibiotics, emerging antimicrobial resistance has become an increasing problem in many pathogens throughout the world. The organisms responsible for bacteraemia vary across geographical boundaries; but few of the pathogens like E. coli, Klebsiella spp., Staphylococcus aureus, Coagulase negative Staphylococci (CoNS), Pseudomonas spp., Salmonella spp. and Acinetobacter spp. are constantly associated with bacteraemia because of their frequent isolation and multidrug resistance which has reached worrying levels. Also some phenotypes such as MRSA, VRE, MR-CoNS, and Carabapenamase producing gram negative organisms such as P.aeruginosa \& Acinetobacter baumanii are of particular concern. Even central venous catheter, invasive devices, surgery and long term stay in ICUs puts the patients at higher risk of BSI.

For practicing physicians, clinical microbiologists and public health officials, knowledge of local antimicrobial resistance patterns is essential to guide empirical and pathogen specific therapy. This information is also critical for optimal decisions regarding formulation of hospital infection control policies, rational public healthcare policies, and national and international research agendas in that area. Antimicrobial resistance surveillance is essential to track changes in microbial populations, estimate the magnitude of the problem and to design and evaluate interventions. However, there is no national level information on resistance among bacteria causing bloodstream infections in India.

Therefore, the present study was a modest attempt with an objective to evaluate the bacteriological profile and tracing resistance among patients with BSI at our hospital. This study also summarizes the strategic resistance patterns of the isolated organisms from the patients admitted in our tertiary care hospital.

\section{Materials and Methods}

This retrospective analysis on blood culturepositive isolates and their antimicrobial susceptibility pattern was carried out during the period starting from 2012-2015 in a tertiary care teaching hospital in southern Rajasthan. Approval for the study was obtained from hospital ethics committee and consent was waived since this is a retrospective evaluation. Only one blood isolate per patient was included in the study. The blood sample were collected in BacT/ALERT FA (adult) and BacT/ALERT PFplus (paediatric) bottles; and received in the department. Inoculated bottles were incubated in BacT/ALERT 3D (Biomerieux) and growth indicated bottles were processed as per company instructions. Identification of microorganisms to species level and antimicrobial susceptibility testing was performed with the automated system vitek 2 compact (Biomerieux).

Statistical analysis was conducted using Microsoft excel 2010. Chi-square test was applied for the comparison of categorical 
variables. $p$ values less than 0.05 were considered as statically significant.

\section{Results and Discussion}

A total of 543 isolates from 4964 blood specimens cultured were analysed during study duration of 2.5 years (2012-2015). The overall culture positivity rate thus obtained was $10.93 \%$. Out of the total isolates analysed, $84.71 \%$ were from intensive care units and rest $15.28 \%$ were from different wards (Table no.I).

The relative distribution frequency of blood isolates is depicted in table no. II. The aerobic or facultative bacterial isolates was found to be $89.50 \%$ and candida species were $10.50 \%$. Among the pathogens; the most common isolates were $27.62 \%$ Coagulase negative staphylococci (CoNS), $25.78 \%$ Escherichia coli, $16.94 \%$ Klebsiella pneumoniae, $\quad 5.89 \% \quad$ Pseudomonas aeruginosa and $5.70 \%$ Non-albicans Candida species.

The resistance pattern to antibiotics for gram negative isolates and gram positive isolates is shown in table no. III and IV respectively.

According to global surveillance reports, bloodstream isolates are the best candidates for the study of antimicrobial susceptibility of human bacterial pathogens. Patients with bacteraemia have remained a treatment challenge. The present study illustrates the BSI bacterial spectrum and antimicrobial resistance pattern in a tertiary care teaching hospital of southern Rajasthan.

During the study period a total 4964 blood samples for aerobic bacterial culture were received in the department for processing, of which 543 were culture positive; with an over all culture positivity rate of $10.93 \%$.Similar rate of blood culture positivity of $12.7 \%$ was also reported by Chand wattal et al.,., from north India. Several studies across India have reported a varied range of positivity ranging from $3.72 \%$ to $44 \%$.The varying rates of blood culture depends upon numerous factors such as the number and amount of blood cultures taken, the system and type of blood culture medium used for bacterial detection. In addition to this most of the patients already received some kind of antibiotics before they come to the tertiary care hospital and self-medication is very common because of the counter availability of medicines (Asmita Ashok, 2016).

In the present study, Gram negative bacteria was found to be responsible for $56.90 \%$ and Gram positive bacteria caused $32.59 \%$ of the BSI. This observation is similar to the various studies done in the patients of developing countries (Vanitha 21-24). In the study conducted by Vanitha et al., had reported $59.1 \%$ of gram negative organisms and $37.7 \%$ gram positive organisms. Similarly Mehta et al.,.., also have reported gram negative organisms as the primary cause of BSI but with a higher rate of $80.96 \%$; whereas only $18 \%$ were gram positives organisms in their study.

In the present study, CoNS were the most commonly isolated Gram positive cocci with an isolation rate of $27.62 \%$, followed by the isolates of $S$. aureus and Enterococci contributing only $2.94 \%$ and $2.02 \%$ of respective isolation rates. In contrast, Asmita Patil et al., and Rakhee baby et al., have reported a higher isolation rate of $27.66 \%$ and $21.46 \%$ for S. aureus. Similar to our CoNS isolation rates; Chand Wattal also have reported 20.3\% in blood. Anu Gupta et al., and Mukherjee et al., also had reported CoNS as most common Gram positive isolate; but with different isolation rates of $9.1 \%$ and $61 \%$ individually. Similar studies 
from other countries carried out by Karlowsky et al., and Japoni et al., had reported $42 \%$ and $67.7 \%$ CoNS respectively. Previously, CoNS isolated from blood culture were considered as contaminants. But in recent years because of the increased use of intra-vascular devices, increase in immunocompromised patients and propensity to form biofilm by the organism; they are now considered as important agent for nosocomial bacteraemia. Meticulous skin disinfection at the time of venepuncture, determination of time to positivity, clinical correlation and appropriate antimicrobial therapy to prevent cross transmission from patient-to- patient can help to differentiate CoNS as potential contaminants or as true pathogens (Cockerill et al., 1997, Kumar Y et al., 2001). Also interpretation of blood cultures that are positive for CoNS require careful reasoning (Anu Gupta, 2010).

Among the Gram negative isolates, E.coli, $(25.78 \%)$ was the primary isolate followed by Klebsiella pneumoniae (16.94\%),P. aeruginosa (5.89\%), and Acinetobacter ssp. $(4.60 \%)$. Similarly E.coli were reported to be most common gram negative bacilli from BSI in many studies. (Vinitha). Kalpesh Gohel et al.,; Wagner et al., also reported E coli as commonest gram negative isolate in BSI. Although Kumar et al., reported predominance of Klebsiella bacteraemia; in the current analysis it was the second most common gram negative organism. This diversity in the frequency can be justified due to the difference in the study plan, geographical location, seasonal variation, hospital infection control policies and disparity of the etiological agents (Asmita Ashok Patil, 2016).

In the present study, Candida spp. accounted for $10.50 \%$ of the BSI pathogens $(5.70 \%$ Non-albicans candida and $4.78 \%$ Candida albicans). This data is comparable to the studies conducted by Anu Gupta et al., (13\%) and Chand Wattal et al., (17.5\%). According to surveillance data from the US centre for disease control and prevention, candida accounts for $12 \%$ of all hospital acquired BSIs (Hidron et al., 2008). The fungal BSI are on rise due to extensive use of antibiotics, aggressive treatment of various diseases, increasing use of invasive devices, an increasing surviving AIDS patients etc. (Anu Gupta, 2010). These all factors in addition to solid organ malignancy, previous surgery, and increase in use of antifungal agents also contributed for the same (Chand Wattal, 2014).

The antimicrobial resistance pattern in the present study for Gram positive isolates was statically significant only for Oxacillin in CoNS isolates during the study period.Anu Gupta et al., also had stated increased methicillin resistant during the two year study periods. This indicates that infections by CoNS may constitute a significant threat to septicemia in our locale and the spectrum of organisms is subject to geographical alterations. We report $100 \%$ sensitivity to vancomycin and linezolid in Staphylococcus aureus; whereas vancomycin resistance of $36.36 \%$ (4/11) in Enterococci was noted with $100 \%$ linezolid sensitivity. Anu Gupta et al., also had reported $16.8 \%$ vancomycin resistance in Enterococci. The possible explanation for this may be due to lower number of isolates of $\mathrm{S}$. aureus in comparison to CoNS; and may be the judicious use of vancomycin and linezolid in our setup.

Among the Enterobactericiae; E. Coli and Klebsiella pneumonia were the commonest to be isolated. Stastical comparison was done between the resistance pattern of isolated strains in the year 2012-2013 and 2014-2015. Statistically significant 
resistance was observed for Ciprofloxacin, Amoxicillin-clavulanic acid, Piperacillintazobactam, and Cefuroxime in E.coli. Imipenem did not show any significant resistance in E.coli during study period. In Klebsiella pneumoniae Amoxicillinclavulanic acid, Piperacillin- tazobactam, and Imipenem were significantly resistant. Anu Gupta et al., also had reported alarming increase of resistance for most of the antibiotics during the study period. Also there was increase in the imipenem resistance in E.coli.

Among the Non-Enterobactericiae; no significant resistance was observed for any antibiotics during the study period. The possible explanation may be the fewer isolates of Pseudomonas species and Acinetobacter species.

Table.1 Distribution of positive blood cultures based on location of the patient (ICU \& Wards)

\begin{tabular}{|l|c|c|c|c|c|}
\hline Location & $\mathbf{2 0 1 2}$ & $\mathbf{2 0 1 3}$ & $\mathbf{2 0 1 4}$ & $\mathbf{2 0 1 5}$ & Total \\
\hline ICUs & 73 & 101 & 133 & 153 & $460(84.71 \%)$ \\
\hline Wards & 17 & 21 & 28 & 17 & $83(15.28 \%)$ \\
\hline
\end{tabular}

Table.2 Year wise distribution of number of blood isolates

\begin{tabular}{|c|c|c|c|c|c|}
\hline Micro-organism & $\begin{array}{l}2012 \\
(n=90)\end{array}$ & $\begin{array}{l}2013 \\
(n=122)\end{array}$ & $\begin{array}{l}2014 \\
(n=161)\end{array}$ & $\begin{array}{l}2015 \\
(n=170)\end{array}$ & $\begin{array}{l}\text { Total } \\
(n=543)\end{array}$ \\
\hline \multicolumn{6}{|l|}{ Gram positive bacteria } \\
\hline CONS & 26 & 32 & 41 & 51 & $150(27.62 \%)$ \\
\hline Staphylococcus aureus & 00 & 04 & 07 & 5 & $16(2.94 \%)$ \\
\hline Enterococcus species & 03 & 02 & 02 & 4 & $11(2.02 \%)$ \\
\hline Total gram positive & 29 & 38 & 50 & 60 & $\begin{array}{l}177 \\
(32.59 \%)\end{array}$ \\
\hline \multicolumn{6}{|l|}{ Gram negative bacteria } \\
\hline Escherichia coli & 30 & 34 & 40 & 36 & $140(25.78 \%)$ \\
\hline Klebsiella pneumoniae & 10 & 21 & 28 & 33 & $92(16.94 \%)$ \\
\hline Acinetobacterbaumanii & 02 & 05 & 09 & 09 & $25(4.60 \%)$ \\
\hline $\begin{array}{l}\text { Pseudomonas } \\
\text { aeruginosa }\end{array}$ & 04 & 07 & 09 & 12 & $32(5.89 \%)$ \\
\hline Others & 03 & 04 & 07 & 06 & $20(3.68 \%)$ \\
\hline Total gram negative & 49 & 71 & 93 & 96 & $\begin{array}{l}309 \\
(56.91 \%)\end{array}$ \\
\hline Candida albicans & 06 & 06 & 08 & 06 & $26(4.78 \%)$ \\
\hline $\begin{array}{lll}\text { Candida } & \text { ssp. } & \text { (Non } \\
\text { albicans) } & & \end{array}$ & 06 & 07 & 10 & 08 & $31(5.70 \%)$ \\
\hline Total fungi & 12 & 13 & 18 & 14 & $57(10.50 \%)$ \\
\hline
\end{tabular}


Table.3 Trend of antimicrobial resistance in Gram positive bacteria

\begin{tabular}{|c|c|c|c|c|c|c|c|c|}
\hline $\begin{array}{l}\text { Organism/ } \\
\text { Antibiotic }\end{array}$ & 2012 & 2013 & 2014 & 2015 & $\begin{array}{l}2012- \\
2013\end{array}$ & $\begin{array}{l}2014- \\
2015\end{array}$ & $P$ value & \\
\hline \multicolumn{9}{|l|}{ CONS } \\
\hline Oxacillin & $12 / 26$ & $31 / 32$ & $45 / 41$ & $47 / 51$ & $43 / 58$ & $92 / 92$ & $<0.0001$ & $\begin{array}{l}\text { Statistically } \\
\text { significant }\end{array}$ \\
\hline Gentamicin & $4 / 26$ & $6 / 32$ & $08 / 41$ & $08 / 51$ & $10 / 58$ & $16 / 92$ & 1 & Not significant \\
\hline Vancomycin & $0 / 26$ & $0 / 32$ & $1 / 41$ & $0 / 65$ & $00 / 58$ & $01 / 92$ & 0.444 & Not significant \\
\hline Linezolid & $0 / 26$ & $0 / 32$ & $1 / 41$ & $1 / 65$ & $00 / 58$ & $02 / 92$ & 0.278 & Not significant \\
\hline \multicolumn{9}{|c|}{ Staphylococcus aureus } \\
\hline Oxacillin & $00 / 00$ & $2 / 4$ & $4 / 7$ & $3 / 5$ & $02 / 04$ & $07 / 12$ & 0.780 & Not significant \\
\hline Gentamicin & $00 / 00$ & $1 / 4$ & $1 / 7$ & $0 / 5$ & $01 / 04$ & $01 / 12$ & 0.369 & Not significant \\
\hline Vancomycin & $00 / 00$ & $00 / 04$ & $00 / 07$ & $00 / 05$ & $00 / 04$ & $00 / 12$ & NA & \\
\hline Linezolid & $00 / 00$ & $00 / 04$ & $00 / 07$ & $00 / 05$ & $00 / 04$ & $00 / 12$ & NA & \\
\hline \multicolumn{9}{|l|}{ Enterococcus } \\
\hline Vancomycin & $00 / 03$ & $01 / 02$ & $01 / 02$ & $02 / 04$ & $01 / 05$ & $03 / 06$ & 0.303 & Not significant \\
\hline Linezolid & $00 / 03$ & $00 / 02$ & $00 / 02$ & $00 / 04$ & $00 / 05$ & $00 / 06$ & NA & \\
\hline
\end{tabular}

Table. 4 Trend of antimicrobial resistance of blood isolates in gram negative bacteria

\begin{tabular}{|c|c|c|c|c|c|c|c|c|}
\hline Organism/antibiotic & 2012 & 2013 & 2014 & 2015 & $\begin{array}{l}2012- \\
2013\end{array}$ & $\begin{array}{l}2014- \\
2015\end{array}$ & $\begin{array}{l}\text { p- } \\
\text { value }\end{array}$ & \\
\hline \multicolumn{9}{|l|}{ E. coli } \\
\hline Amikacin & $06 / 30$ & $5 / 34$ & $4 / 40$ & $7 / 36$ & $11 / 64$ & $11 / 76$ & 0.623 & Not significant \\
\hline Ciproflaxacin & $20 / 30$ & $28 / 34$ & $36 / 40$ & $33 / 36$ & $48 / 64$ & $69 / 76$ & $\mathbf{0 . 0 1 8}$ & Statistically significant \\
\hline Amoxi-clav & $04 / 30$ & $6 / 34$ & $14 / 40$ & $17 / 36$ & $10 / 64$ & $31 / 76$ & 0.001 & Statistically significant \\
\hline Piperacillin+Tazobactam & $02 / 30$ & $05 / 34$ & $08 / 40$ & $12 / 36$ & $07 / 64$ & $20 / 76$ & 0.015 & Statistically significant \\
\hline Cefuroxime & $02 / 30$ & $4 / 34$ & $08 / 40$ & $09 / 36$ & $06 / 64$ & $17 / 76$ & $\mathbf{0 . 0 3 6}$ & Statistically significant \\
\hline Imipenem & $0 / 30$ & $0 / 34$ & $1 / 40$ & $1 / 36$ & $00 / 64$ & $02 / 76$ & 0.255 & Not significant \\
\hline \multicolumn{9}{|l|}{ Klebsiella } \\
\hline Amikacin & $4 / 10$ & $08 / 21$ & $11 / 28$ & $11 / 33$ & $12 / 31$ & $22 / 61$ & 0.850 & Not significant \\
\hline Ciproflaxacin & $08 / 10$ & $17 / 21$ & $21 / 28$ & $26 / 33$ & $25 / 31$ & $47 / 61$ & 0.742 & Not significant \\
\hline Amoxi-clav & $2 / 10$ & $4 / 21$ & $11 / 28$ & $16 / 33$ & $06 / 31$ & $27 / 61$ & 0.017 & Statistically significant \\
\hline Piperacillin+Tazobactam & $2 / 10$ & $4 / 21$ & $14 / 28$ & $19 / 33$ & $06 / 31$ & $33 / 61$ & 0.001 & Statistically significant \\
\hline Cefuroxime & $1 / 10$ & $2 / 21$ & $4 / 28$ & $6 / 33$ & $03 / 31$ & $10 / 61$ & 0.355 & Not significant \\
\hline Imipenem & $0 / 10$ & $1 / 21$ & $8 / 28$ & $11 / 33$ & $01 / 31$ & $19 / 61$ & 0.002 & Statistically significant \\
\hline \multicolumn{9}{|l|}{ Acinetobacter } \\
\hline Amikacin & $1 / 2$ & $3 / 5$ & $4 / 9$ & $5 / 9$ & 04/07 & 9/18 & 0.753 & Not significant \\
\hline Gentamicin & $1 / 2$ & $1 / 5$ & $1 / 9$ & $1 / 9$ & 02/07 & 02/18 & 0.294 & Not significant \\
\hline Ciproflaxacin & $1 / 2$ & $3 / 5$ & $6 / 9$ & $6 / 9$ & 04/07 & $12 / 18$ & 0.674 & Not significant \\
\hline Imipenem & $1 / 2$ & $1 / 5$ & $3 / 9$ & $4 / 9$ & 02/07 & $07 / 18$ & 0.638 & Not significant \\
\hline Piperacillin+Tazobactam & $1 / 2$ & $2 / 5$ & $4 / 9$ & $5 / 9$ & 03/07 & $09 / 18$ & 0.719 & Not significant \\
\hline \multicolumn{9}{|l|}{ Pseudomonas } \\
\hline Amikacin & $2 / 4$ & $2 / 7$ & $1 / 9$ & $2 / 12$ & $04 / 11$ & $03 / 21$ & 0.150 & Not significant \\
\hline Ceftazidime & $2 / 4$ & $3 / 7$ & $2 / 9$ & $2 / 12$ & $05 / 11$ & $04 / 21$ & 0.119 & Not significant \\
\hline Ciproflaxacin & $2 / 4$ & $1 / 7$ & $3 / 9$ & $3 / 12$ & 03/11 & $06 / 21$ & 0.952 & Not significant \\
\hline Piperacillin+Tazobactam & $0 / 5$ & $1 / 7$ & $1 / 9$ & $2 / 12$ & $01 / 11$ & $03 / 21$ & 0.682 & Not significant \\
\hline Imipenem & $1 / 5$ & $2 / 7$ & $2 / 9$ & $3 / 12$ & 03/11 & $05 / 21$ & 0.802 & Not significant \\
\hline
\end{tabular}

One of the important outcomes of the present study was the relief for intensivist that resistant level of life saving drugs like Meropenem and Imipenem has not yet 
increased to alarming stage. Thus these antimicrobials can be used as an early commencement treatment and later deescalate which can plays a vital role in reducing morbidity and mortality in BSI. The basis for this early treatment is the information about the likely pathogen and its antibiotic resistance pattern. Present study provides much needed information on the prevalence and antibiotic sensitivity pattern of prevalent blood pathogens. The analysis of resistance will help in formulating antibiotic policy and to decide the vacation period for any antibiotic in particular if required. The data will also help in limiting the indiscriminate use of antibiotics (Asmita et al., 2016). The main forces driving the increase in antimicrobial resistant bacteria are poor infection control practices and inappropriate use of antibiotics. Specific antibiotic utilization strategies like antibiotic restriction, combination therapy, and antibiotic recycling may help to decrease or prevent the emergence of resistance. Specific usage based on susceptibility testing may also reduce the incidence of BSIs (Vanitha).

In the current study, we also acknowledge several limitations to our study. First this is a single centre study and may not reflect the true status of the antimicrobial pattern. Second, the duration of the study is short and needed to be extended to get a prolific result. Though the fungaemia was seen due to Candida isolates (non-albicans), data from antifungal susceptilibility could be added benefit.

In conclusion, the present study revealed that Gram negative bacilli i.e. E. coli and Klebsiella pneumoniae predominantly an leading cause of BSI in our tertiary care setup; followed by CoNS. The significance of CoNS bacteraemia should be evaluated better in light of clinical profile of patient. In present study, increased resistance was observed in CoNS for oxacillin; an alarming increase of antibiotic resistance for various antibiotics was noted for Klebsiella pneumoniae during study period. E.coli showed significant resistant to Ciprofloxacin, Amoxicillin-clavulanic acid, Piperacillin- tazobactam, and Cefuroxime in E.coli with no increase in carbapenems resistance. This calls for implementation of strict antibiotic prescribing policies and hospital infection control guidelines. Ongoing surveillance for antimicrobial susceptibility remains essential in case of BSI and will enhance efforts to identify resistance and attempt to prevent its spread.

\section{Acknowledgement}

Authors acknowledge the immense help received from the scholars whose articles are cited and included in references of this manuscript. The authors are also grateful to authors/editors/publishers of all those articles, journals and books from where the literature for this article has been reviewed and discussed.

\section{References}

Anu Gupta, Shweta Sharma, Anita Arora, Ashish Gupta; "Changing trends of in vitro antimicrobial resistance patterns in blood isolates in a tertiary care hospital over a period of 4 years"; Indian J. Med, Sci., Volume : 64, Issue : 11, Page : 485492.

Asmita Ashok Patil, Pratibha, J. Dalal. " Bacterial profile and resistance pattern of bacterial isolates from blood culture - a five year study in tertiary care teaching hospital", European J. Pharma. Med. Res., 3(4): page: 373-377.

Butt, T., Afzal, R.K., Ahmad, R.N., Salman, M., Mahmood, A., Anwar, M. 2004. Blood stream infections in febrile neutropenic patients: Bacterial spectrum and anti microbial susceptibility pattern. J. Ayub. 
Med. Coll. Abbottabad., 16:18-22.

Chand Wattal, Reena Raveendrana, NeerajGoela, Jaswinder Kaur Oberoi, Brijendra Kumar Raob. 2014. Ecology of blood stream infection and antibiotic resistance in intensive care unit at a tertiary care hospital in North India, The Brazilian J. Infect. Dis., 1 vol. 8(3): page 245-251.

Chen, L.y, Tang, J.L., Hsueh, P.R., You. M., Huang, S.Y., Chen, Y.c, et al., 2004. Trends and anti microbial resistance of pathogens causing blood stream infections among febrile neutropenic adults with hematological malignancy. $J$. Formos Med. Assoc., 103: 526-32.

Cockerill, F.R., Reed, G.S., Hughes, J.G., et al., 1997. Clinicalcomparison of BACTEC 9240 plus aerobic/F resin bottles andthe isolator aerobiac culture system for detection of bloodstream infections. $J$. Clin. Microbiol., 5: 1469-72.

FigueraEsparaza, M., Carballo, M., Silva, M., Figuerdo, A., Avilan, J. 2006. Microbiological isolates in patients with febrile neutropenia and nematological neoplasias. Rev. Esp. Quimioter, 19: 24751.

Hidron, A.I., Edwards, J.R., Patel, J., et al., 2008. Antimicrobial- resistant pathogens associated with healthcare-associated infections: annual summary of data reported to the National Healthcare Safety Network at the Centers for Disease Control and Prevention 2006-2007. Infect. Control Hosp. Epidemiol., 29: 996-1011.

Japoni, A., Vazin, A., Hamedi, M., et al., 2009. Multidrug-resistant bacteria isolated from intensive-care-unit patient samples. Braz. J. Infect. Dis., 13: 118-229.

Karlowsky, J.A., Jones, M.E., Draghi, D.C., Thornberry, C., Sahm, D.F., Volturo,
G.A. 2002. Prevalence and antimicrobial susceptibilities ofbacteria isolated from blood cultures of hospitalized patientsin the United States in 2002. Ann. Clin. Microbiol. Antimicrob., 3: 1-8.

Kumar, Y., Qunibi, M., Neal, T.J., et al., 2001. Time to positivity ofneonatal blood cultures. Arch. Dis. Child Fetal Neonatal Ed., 85: 182-6.

Kumar, S.M., Razvi, S., Vidhani, V.K., Sharma. 2004. Changing face of septicemia and increasing drug resistance in blood isolates. Indian J. Pathol. Microbiol., 47: 441- 446.

Mukherjee, T., Pramod, K., Gita, S., Medha, Y.R. 2005. Nosocomial infections in geriatric patients admitted in ICU. J. of Ind, Acad. of Geriatircs, 2: 61-64.

Nimra, L.F., Batchoun, R. 2004. Communityacquired bacteraemia in a rural area: predominant bacterial species and antibiotic resistance. J. Med. Microbiol., 53: 1045-49.

Raakhee baby thananki, Hemaprakashkumari and s. subbarayudu. 2014. "Danger in the blood" BSI and current trend in antimicrobial resistance; Int. J. Pharma and Bio Sci., 5(1): (B) $827-834$

Vanitha rani, N., Kannan gopal, Venkatanarendra, M., Vishwakanth, D., V.R.D. Nagesh, Yogitha, M., Venkatasunil, M., Thennarasupalani. A retrospective study on blood stream infections and antibiotic susceptibility patterns in a tertiary care teaching hospital; Int. J. Pharmacy and Pharma. Sci., Vol 4, Issue 1, page 543-548.

Wagner, G.E. 1990. Bacteremia and septicemia. In: Kingsbury, D.T., and Wagner. G.E.(eds.) Microbiology. $2^{\text {nd }}$ edition. John Wiely \& Sons. Inc., USA. Pp.315-320.

\section{How to cite this article:}

Shweta Bohra, Anamika Vyas, Mrityunjay Kumar and A.S. Dalal. 2016. Secular Trend of Antibiotic Resistance in Blood Stream Infections-A retrospective Analysis. Int.J.Curr.Microbiol.App.Sci. 5(6): 798-805.

doi: http://dx.doi.org/10.20546/ijcmas.2016.506.089 